\title{
Changes in diatom patch-size distribution and degradation in a spatially self-organized intertidal mudflat ecosystem
}

\author{
E. J. Weerman, ${ }^{1,2,6}$ J. Van Belzen, ${ }^{1}$ M. Rietkerk, ${ }^{3}{ }^{\text {S. Temmerman, }}{ }^{4}$ S. Kéfi, ${ }^{5}$ P. M. J. Herman, ${ }^{1}$ and J. Van de Koppel ${ }^{1}$ \\ ${ }^{1}$ Centre for Estuarine and Marine Ecology, Netherlands Institute of Ecology (NIOO-KNAW), P.O. Box 40, 4400 AC Yerseke, \\ The Netherlands \\ ${ }^{2}$ Aquatic Ecology and Ecotoxicology (IBED), University of Amsterdam, P.O. Box 94240, 1090 GE Amsterdam, The Netherlands \\ ${ }^{3}$ Department of Environmental Sciences, Utrecht University, P.O. Box 80115, 3508 TC Utrecht, The Netherlands \\ ${ }^{4}$ University of Antwerpen, Department of Biology, Universiteitsplein 1, 2610 Wilrijk, Belgium \\ ${ }^{5}$ Institut des Sciences de l'Evolution, CNRS UMR 5554, Bat 22, second floor, Université de Montpellier II, CC 065, 34095 Montpellier
} Cedex 05, France

\begin{abstract}
Self-organized spatial patterns have been proposed as possible indicators for regime shifts in ecosystems. Until now, this hypothesis has only been tested in drylands. Here, we focus on intertidal mudflats where regular spatial patterns develop in early spring from the interaction between diatom growth and sedimentation but disappear when benthic herbivore abundance increases in early summer, accompanied by a dramatic shift to a bare mudflat. We followed the patch-size distributions of diatom biofilms during this degradation process. As time progressed, we found a temporal change in the spatial configuration occurring simultaneously with the loss of the diatom-sediment feedback. This indicates a gradual failure in time of the self-organization process that underlies regular patterning in this ecosystem. The path to degradation co-occurred with the loss of the larger patches in the ecosystem, which resulted in a decrease of the truncation in the patch-size distribution. Hence, our study in mudflat ecosystems confirms the general hypothesis that spatial patterns can provide important clues about the level of degradation. Nevertheless, our study highlights the need for thorough study about the type of spatial patterns and the nature of the underlying feedbacks before a reliable assessment of ecosystem status can be made, as changes in patchsize distribution differed markedly with those observed in other ecosystems.
\end{abstract}

Key words: diatom-sediment feedback; herbivory; intertidal mudflats; regime shifts; scale-dependent feedbacks; spatial patterns.

\section{INTRODUCTION}

Self-organized spatial patterns have been described in a range of terrestrial and aquatic ecosystems (Pascual and Guichard 2005, Rietkerk and Van de Koppel 2008). These patterns develop from strong feedback mechanisms that can generate coherent spatial patterns at a large spatial scale, ranging from regular to irregular patterns depending on the underlying mechanisms (Pascual and Guichard 2005, Rietkerk and Van de Koppel 2008). Recently, selforganized spatial patterns have been put forward as potential indicators for ecosystem degradation in response to changing levels of environmental or biotic stress in arid ecosystems (Rietkerk et al. 2004). In sub-Saharan drylands, a combination of low rainfall and scale-dependent feedback between vegetation and its limiting resource, water, can induce regular, self-organized spatial vegetation patterns (Barbier et al. 2008). Decreases in rainfall are predicted to induce a sequence of changes in these spatial patterns, the breakdown of regular patterns in smaller

Manuscript received 5 April 2011; revised 20 September 2011: accepted 29 September 2011: final version received 4 November 2011. Corresponding Editor: J. J. Stachowicz.

${ }^{6}$ E-mail: ellenweerman@ gmail.com patches, before the ecosystem collapses to a degraded state (Rietkerk et al. 2004). In Mediterranean ecosystems, a local facilitation mechanism due to the ameliorating effect of the vegetation on its local abiotic environment is proposed to generate self-organized spatial patterns. These feedbacks can result either in spatial patterns with a dominant patch size, characterized by a truncated power law (Maestre and Escudero 2009, Kéfi et al. 2007, Lin et al. 2010), or in irregular spatial patterns, characterized by a power law patch-size distribution (Kéfi et al. 2007). These studies investigated whether predictable changes in the statistical characteristics of spatial vegetation patterns could be used as indicators for ongoing degradation to a desert state. It was suggested that when a Mediterranean dryland becomes more degraded, because, e.g., of overgrazing, the patch-size distribution progressively loses its larger patches and becomes more truncated (Kéfi et al. 2007; but see discussion in Maestre and Escudero 2009, Kéfi et al. 2010, Maestre and Escudero 2010). Since desertification might involve tipping points at unknown thresholds and is often difficult to reverse, the development of indicators based on spatial patterns could be important for ecosystem management to predict when catastrophic change is looming. 
Due to the fact that the search for predictable changes in characteristics of self-organized spatial patterns has focused on vegetation patchiness in drylands (Rietkerk et al. 2004, Kéfi et al. 2007, 2011, Guttal and Jayaprakash 2009, Maestre and Escudero 2009, Lin et al. 2010, von Hardenberg et al. 2010, Pueyo 2011), application of this theory is restricted to semiarid ecosystems. Moreover, most studies tested changes in patch-size distributions by comparing spatially distinct systems along a gradient of decreasing rainfall or increasing grazing intensity (Kéfi et al. 2007, Maestre and Escudero 2009, Lin et al. 2010). When comparing spatially distinct ecosystems, the environment may vary, introducing possible confounding factors. Whether these patterns also show consistent changes preceding a temporal shift to a degraded state remains unstudied. Hence, the hypothesis that selforganized patterns show a coherent, predictable change in spatial structure when developing toward a degraded state would benefit from further testing in other ecosystems, including the explicit analysis of time series of how spatial patterns change in time.

In this paper, we describe temporal changes in the spatial configuration of benthic diatom patterns on the Kapellebank intertidal mudflat in the Netherlands. Regular spatial patterns consisting of diatom-covered hummocks alternating with water-filled hollows develop each spring (Blanchard et al. 2000, de Brouwer et al. 2000, Lanuru et al. 2007; see Plate 1), due to interactions between diatom growth and geomorphological processes (Weerman et al. 2010). The development of spatial patterns emerging from the stress-divergence mechanism has been described in a wide range of ecosystems, e.g., boreal forests, wetlands, and intertidal mudflats in spatial patterns (Hiemstra et al. 2002, Larsen et al. 2007, Saco et al. 2007, Temmerman et al. 2007, van Wesenbeeck et al. 2008). On our intertidal mudflat, $80 \%$ of the area is covered with regular spatial patterns. In the remaining $20 \%$ of the study area, an irregular patchy structure of benthic diatoms is visible, but bed level differences are absent, suggesting the lack of strong feedback mechanisms in these areas. On the whole study area, as time progresses, a gradual and seasonal increase in benthic herbivory causes a sudden change from a spatially patterned intertidal flat with high diatom biomass to a topographically homogeneous sediment at around early June each year (Weerman et al. 2011). The temporal scale of spatial pattern formation in mudflats is, thus, much smaller than the temporal scale of spatial patterns in drylands. In drylands, shifts between desert and vegetated state occur at very long, geological time scales. On intertidal mudflats, spatial patterns degrade each year when macrobenthos biomass increases; in the next spring, when macrobenthos biomass is low, spatial patterns can develop again. Hence, this intertidal mudflat ecosystem provides unique characteristics that make it an extremely interesting case study: (1) the presence of spatial patterns, both regular and irregular, and (2) the buildup and collapse of the system, which can be observed at short time scales and is repeated every year.

Here, we investigate the hypothesis that temporal changes in patch-size distributions precede degradation in spatially self-organized mudflat ecosystems. To this end, we took aerial photographs during the season, starting when spatial patterns were clearly visible (April) until the period when spatial patterns had degraded due to high benthic herbivore numbers (June). We subsequently analyzed the patch-size distributions of observed diatom patches from the classified aerial photographs. Since, in undisturbed conditions, diatom-covered mudflats form regular patterns (Weerman et al. 2010) characterized by a dominant patch size, we expect that with increasing grazing pressure the patch-size distribution would change from a truncated to a less truncated power law as disturbance by grazing increases and fragments the patches. Furthermore, we studied how changes in other signatures that reflect the feedback processes underlying spatial self-organization in our system (e.g., diatomsediment feedbacks) changed with increased grazing pressure. Finally, we put these results in the broader context of the literature on spatial indicators.

\section{Materials and Methods}

\section{Study site and experimental setup}

A field study was performed at the Kapellebank, a tidal flat along the edges of the Westerschelde estuary, in the Netherlands $\left(51^{\circ} 27^{\prime} \mathrm{N}, 3^{\circ} 58^{\prime} \mathrm{E}\right)$. Each year, from early spring until the onset of summer, a regular spatial self-organized pattern of diatom-covered hummocks and water-filled hollows are observed on this intertidal flat (Appendix A). These patterns develop when the interaction between the accumulation of sediment on the hummocks and drainage of water toward the hollows generates a scale-dependent feedback of local-scale facilitation and longer range inhibition of diatom growth, providing an explanation for regular spatial pattern formation when herbivore densities are low (Weerman et al. 2010). Similar regular spatial diatom patterns have been described in other intertidal mudflats (Christie et al. 2000, de Brouwer et al. 2000, Le Hir et al. 2000, Paterson et al. 2000, Lanuru et a1. 2007). In our system, the diatom patterns have a strong seasonality and face temporal variability in hydrodynamic conditions throughout the season (de Brouwer et al. 2000, Weerman et al. 2011).

Six $12 \times 12 \mathrm{~m}$ plots were marked on the Kapellebank, in which sediment bed level and diatom cover were determined in April, May, and June 2008. Three plots were chosen in an area with regular hummock-hollow structure (hereafter referred to as the "regular plots"); three control plots were chosen in an area on flat sediment lacking regular spatial patterns (hereafter referred to as the "irregular plots"). We analyzed the six plots for spatial patterns of benthic algae and sediment bed level during these months. 


\section{Spatial patterns of benthic algae}

Aerial photographs for pattern analysis were taken with a digital camera (Sony Cybershot DSC-V3; Sony Electronics. San Diego, California, USA) attached to a helium-inflated blimp-shaped balloon (Floatograph Technologies, Silver Spring, Maryland, USA), which was attached to a tether line. Photographs were obtained from approximately $50 \mathrm{~m}$ height covering an area of approximately $50 \times 40 \mathrm{~m}(3072 \times 2304$ pixels $)$. The corners of the plots were marked with a colored marker that was used to locate the plots during the image processing.

\section{Landscape morphology}

To collect morphological data of the landscape in the six plots a Riegl LSM Z-210 3D laser scanner (Riegl Instruments, Horn, Austria) was used and controlled by the software RiScan Pro (Riegl Instruments). This laser scanner collects data points of $x, y, z$ coordinates of the sediment surface, using an infrared laser beam emitted in precisely defined angular directions controlled by a spinning mirror arrangement. Prior to the scans, five reflectors were placed around the plot, the position of these reflectors was measured using a DGPS (Thales Group, Neuilly-sur-Seine, France) and these were automatically located by the RiScan Pro software, which used the DGPS data for georeferencing the data points measured by the laser.

\section{Data analysis}

Indicators for spatial self-organization.-First, we analyzed the regularity of the diatom patterns using spatial autocorrelation (Moran $I$ ) of the diatom patches (Legendre and Legendre 1998). Similar to Weerman et al. (2010), we analyzed the intensity of the blue channel within the RGB images, since this corresponds to diatom biomass most closely, and therefore these pixel values were used for subsequent analysis. We applied a two-dimensional anisotropic matrix implementation, which calculates a correlogram for four major directions $(0,45,90$, and 135 degrees) in Matlab 2007b (MathWorks, Natick, Massachusetts, USA). The algorithm is constrained to relatively small images because of its memory use. We selected a square of $75 \times 75$ pixels (corresponding to $3 \times 3 \mathrm{~m}$ ) in the middle of each plot for autocorrelation analysis. Of the four directional correlograms, we selected the one with the highest correlation for interpretation. This was, in all cases, the correlogram with a direction perpendicular to the main direction of the water current. High positive autocorrelation corresponds to more similar diatom biomass at a certain distance, while dissimilarity results in negative autocorrelation (Legendre and Legendre 1998). Second, we investigated the underlying mechanisms of these patterns by checking for the presence of positive feedbacks. Therefore, we generated a digital elevation matrix for each plot from the landscape morphology data measured with the laser scanner. After fixing the corners of these plots by using their DGPS coordinates, the points from the Riegl laser scanner were interpolated into $300 \times$ 300 matrix, where each pixel corresponds to $4 \mathrm{~cm}$, using a nearest-neighbor interpolation. Similarly, the aerial photographs were rescaled into the same resolution of the laser scanner data of $300 \times 300$ pixels using a sliding neighborhood filter in Matlab 2007b. Since diatom content is based on the intensity of the blue channel in the RGB pictures, we calculated the relative diatom content as difference from the average intensity of the blue channel in each picture. We then analyzed the correlation between relative diatom concentration and sediment bed level of 3000 randomly selected points of the aerial photographs and landscape morphology data images using Pearson's correlation coefficient. When scale-dependent feedbacks are intact, we expect high diatom content on top of the hummocks and low diatom content in the hollows. Therefore, a positive correlation between diatoms and sediment bed indicates a functional scale-dependent feedback between diatoms and sediment (Weerman et al. 2010).

Patch-size distribution.-From the raw aerial photographs, the $12 \times 12 \mathrm{~m}$ plots were selected and a binary image was created from each plot by maximum likelihood classification on the RGB pictures. This method was used to classify the pixels for each photograph into two classes: diatoms or bare sediment. The classes were based on manually selected pixels $(n=$ 15 pixels) in each class from which the brightness in the red, green, and blue band was recorded and extrapolated to the other pixels in the image. For each picture, the classification method was checked by comparing the binary images with the raw photographs (Appendix B). From this binary image, the cover fraction was calculated and patches were assigned using a Moore neighborhood of eight adjacent pixels. A minimal patch size was set at four pixels to avoid artifacts at patch edges affecting patch-size distributions. For each unique patch size, the number of occurrences was calculated. From this vector of patch sizes and their occurrences, the inverse cumulative distribution was calculated and an upper-truncated power law was fitted to the inverse cumulative distribution according to Burroughs and Tebbens (2001). Note that it differs from the fitting procedure typically used in previous studies for noncumulative patch-size distributions, e.g., by Kéfi et al. (2007) or Maestre and Escudero (2009). These authors used a binning-based method but this takes away the information about the patch-area distribution within each bin (White et al. 2008). Therefore we used a cumulative distribution in which no binning is used. The patch-size distributions were fitted using the following equation describing an upper-truncated cumulative number-size distribution based on a power law (Burroughs and Tebbens 2001):

$$
M(r)=C\left(r^{-\gamma}-r_{\mathrm{T}}^{-\gamma}\right)
$$

where $\mathrm{M}(r)$ is the cumulative fitting function of patches of size $r$ (in $\mathrm{cm}^{2}$ ), $C$ is a constant, $\gamma$ is the scaling 
A) Regular patterns, April

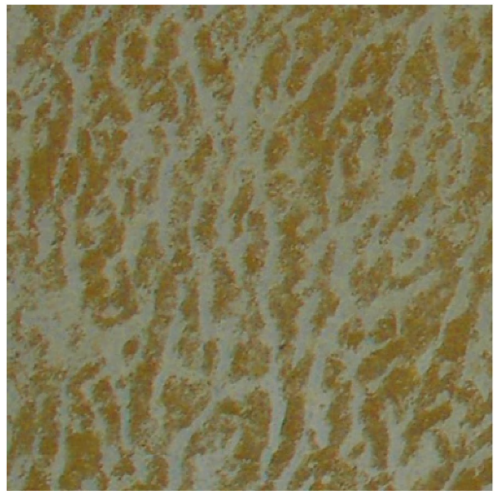

D) Irregular patterns, April

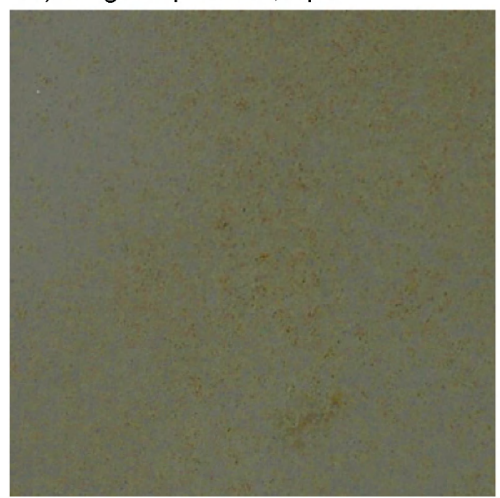

B) Regular patterns, May

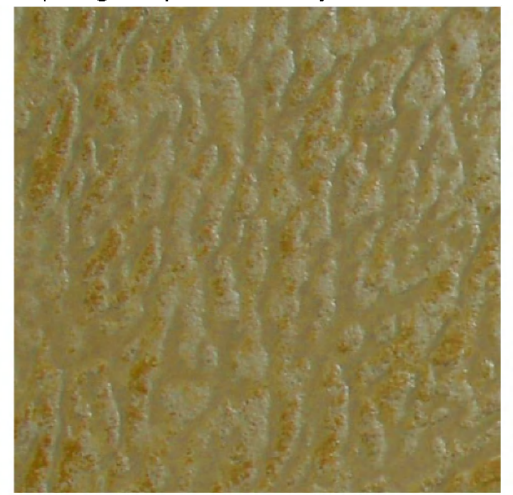

E) Irregular patterns, May

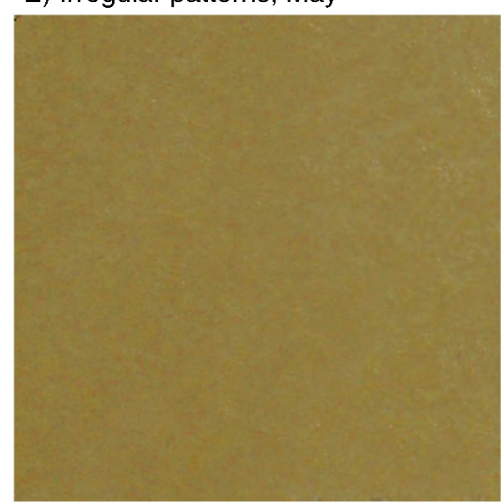

C) Regular patterns, June

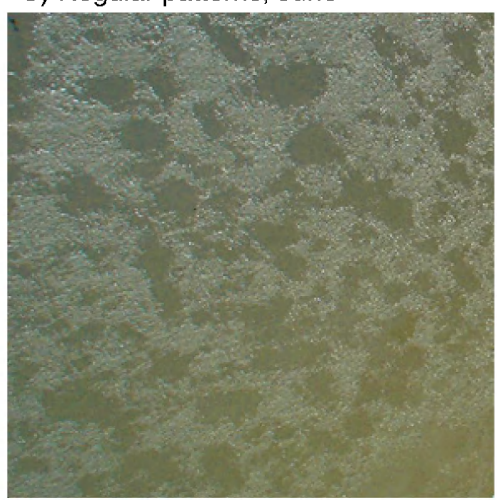

F) Irregular patterns, June

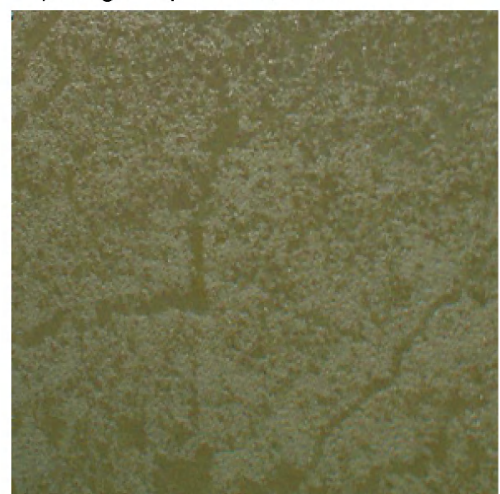

FIG. 1. Aerial photographs of plots in April. May, and June for the location with regular spatial patterns (A-C) and the location with irregular spatial patterns (D-F). Brown areas correspond to diatom abundance while gray areas correspond to bare sediment. Plot size is $12 \times 12 \mathrm{~m}$. See Appendix B for the binary images.

exponent, and $r_{\mathrm{T}}$ is the patch size at which the truncation occurs. For the fitting procedure, we used a pseudo-random search algorithm (pricefit module), which is provided in the $\mathrm{R}$ package ecolMod (Soetaert and Herman 2009). Appendix A shows that, although this method is sensitive to the size of the plots, the truncation is determined by the size of the observed patches, rather than an artifact of plot size.

Kéfi et al. $(2007,2011)$ suggested that the patch-size distribution of vegetation in Mediterranean ecosystems should switch from a power law to a truncated power law when the ecosystem is approaching desertification. A recent paper of Pueyo (2011) showed that, for fundamental reasons, we should move away from the power law vs. truncated power law dichotomy to other categorizations. More precisely, a truncated power law can be fitted to all the data sets (if taken at a large enough scale) and we expect that truncation becomes stronger when a dryland approaches degradation (following similar reasoning as Kéfi et al. [2007]). In line with this, all our patch-size distributions were fitted using Eq. 1. To evaluate the degree of the truncation of the curves, we defined the observed truncation to be the ratio between the intercept $\mathrm{M}(r)$ of the full truncated power law model and of the power law model without truncation, but with similar scaling exponent (Appendix A). The observed truncation is a measure of the degree of bending independent of where the bending occurs. Its calculation involves comparing the fitted truncated power law model with a pure power law model that uses the scaling exponent from the prior, truncated fit. Note that this "pure" power law does not characterize a "healthy" reference state, as was assumed in Kéfi et al. 2007, but is merely derived to calculate the degree of bending within the reported data range. Differences in cover, average patch size, the scaling exponent $(\gamma)$, the patch size at which the truncation occurs $(r T)$, and the observed truncation between different sampling times were analyzed using Students' paired $t$ test (two-tailed).

\section{RESULTS}

In both regular and irregular patterned plots, the cover fraction of diatoms decreased significantly between April and May (Figs. 1 and 2A; regular, $t_{2}=$ 20.29, $P<0.01$; irregular, $t_{2}=8.04, P<0.05$ ). This degradation co-occurred with a decrease in average patch size in the regular patterned plots (Fig. 2B; $t_{2}=$ 12.34, $P<0.01$ ) while patch sizes in the irregular patterned plots did not change (Fig. $2 \mathrm{~B} ; t_{2}=4.00, P>$ 0.05 ). In June, no diatom patches could be detected, and 

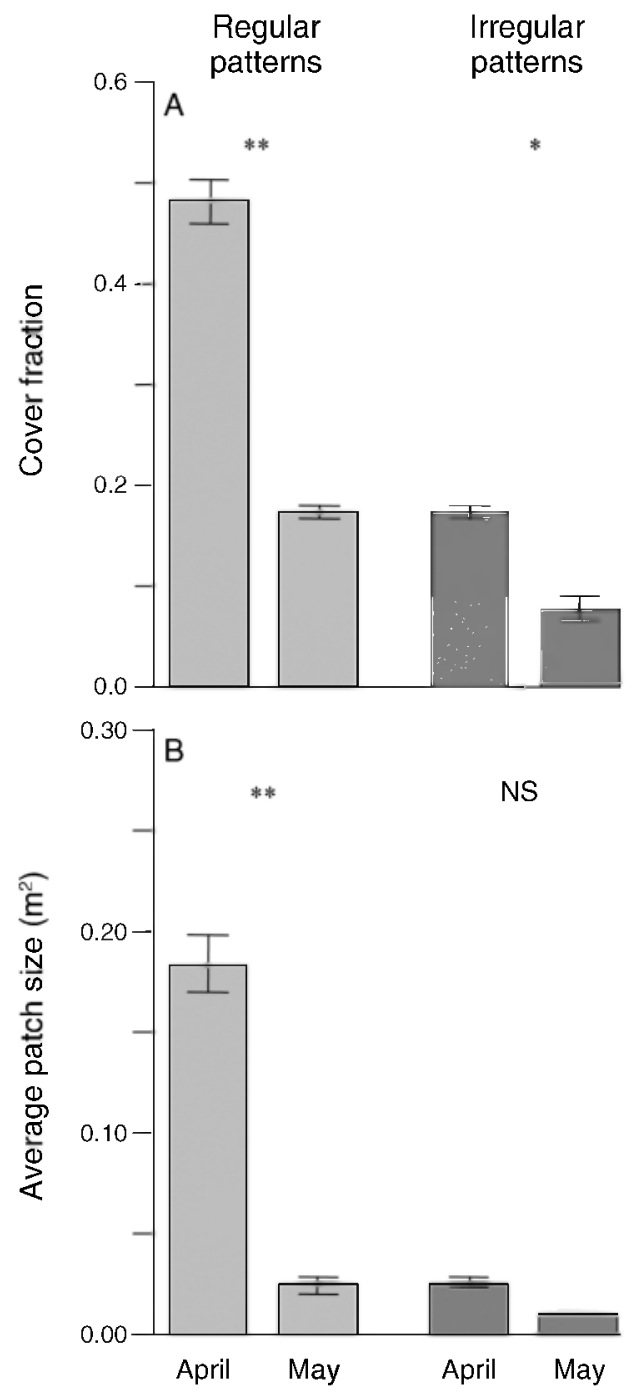

FIG. 2. (A) Cover fraction and (B) average patch size of the irregular and regular plots in April and May. Light gray bars represent the regular spacing patterns, and dark gray bars represent the irregular patterns $(n=3$; error bars indicate $\pm \mathrm{SE})$.

* $P<0.05$; * $P<0.01$ * ** $P<0.001$.

therefore we argue that the ecosystem can be considered as completely degraded. In the rest of this paper, we thus focus on the analysis of the patterns in April and May.

Aerial photographs of the plots revealed clear visual differences between the regular and irregular plots (Fig. 1), which were confirmed by autocorrelation analysis. The regular plots showed positive autocorrelation up to $20 \mathrm{~cm}$ lag distance, followed by significant negative autocorrelation from 20 to $80 \mathrm{~cm}$ (Fig. 3A). This confirms that, in these plots, bands of high diatom biomass were regularly distributed in the direction perpendicular to the flow direction with a wavelength of roughly $1 \mathrm{~m}$. In May, this regularity was lost, since only positive Moran $I$ values were found (Figs. 1B and 3B). The irregular plots showed only positive Moran I values in both April and May, indicating a lack of regularity in these plots (Fig. 3C and D).

To investigate possible feedbacks underlying the observed patterns, we looked at the correlation between diatom abundance (as estimated from the aerial photographs) and sediment bed-level elevation in April and May (Fig. 4). In the regular plots, a strong positive correlation was found in April (Fig. 4A), while the correlation was absent or very weak in May (Fig. 4B). This suggests that the change in spatial configuration observed in the regular plots co-occurred with the disappearance of the scale-dependent feedback between diatom growth and sedimentation processes. In the irregular plots, no or very weak correlation was found in both April and May (Fig. 4C and D), which suggests that no scale-dependent feedbacks were underlying the observed irregular spatial patterns.

Consistently with the regularity of the patterns revealed by autocorrelation analysis, the patch-size distributions showed consistent changes. The regular plots in April were characterized by an excess of patches of intermediate size than can be expected based on a pure power law. This suggests the presence of a dominant spatial scale, which is a characteristic of regular patterns (Fig. 5A). The other distributions (regular plots in May and irregular plots in April and May) approached a power law distribution (Fig. 5B-D). For the regular plots, the scaling exponent $(\gamma)$ was found to be higher in May compared to April (Fig. 6A; regular, $\left.t_{2}=-9.13, P=0.05\right)$. The same trend was observed in the irregular plots, but was not significant (Fig. $6 \mathrm{~A} ; t_{2}=$ $-2.88, P \geq 0.05$ ). At the same time, we observed a clear decrease of the truncation parameter in the regular plots between April and May (Fig. 6B, $t(2)=22.27, P<0.01$ ), while this was not significant (but also decreasing) in the irregular plots (Fig. 6B; $t_{2}=1.73, P>0.05$ ). Looking more into the details of the distribution shapes, the observed truncation was significantly different for regular plots between April and May (Fig. 6C; $t_{2}=$ $12.02, P<0.01$ ), which indicates that the degree of truncation decreases, as degradation increases. The irregular plots did not show any significant change between these months (Fig. $6 \mathrm{C} ; t_{2}=2.34, P>0.05$ ).

\section{Discussion}

Recent studies have proposed that changes in the spatial configuration of self-organized vegetation patterns in drylands can serve as possible indicators of ecosystem degradation (Rietkerk et al. 2004, Kéfi et al. 2010). To investigate if this applies to other ecosystems as well, we studied the changes in spatial patterns of diatoms during the degradation process on intertidal mudflats (Weerman et al. 2011). Our results showed consistent changes in the patch-size distribution of the self-organized regular patterns as the disturbance, caused by herbivores, increased. This is generally in agreement with the idea that the spatial organization of 

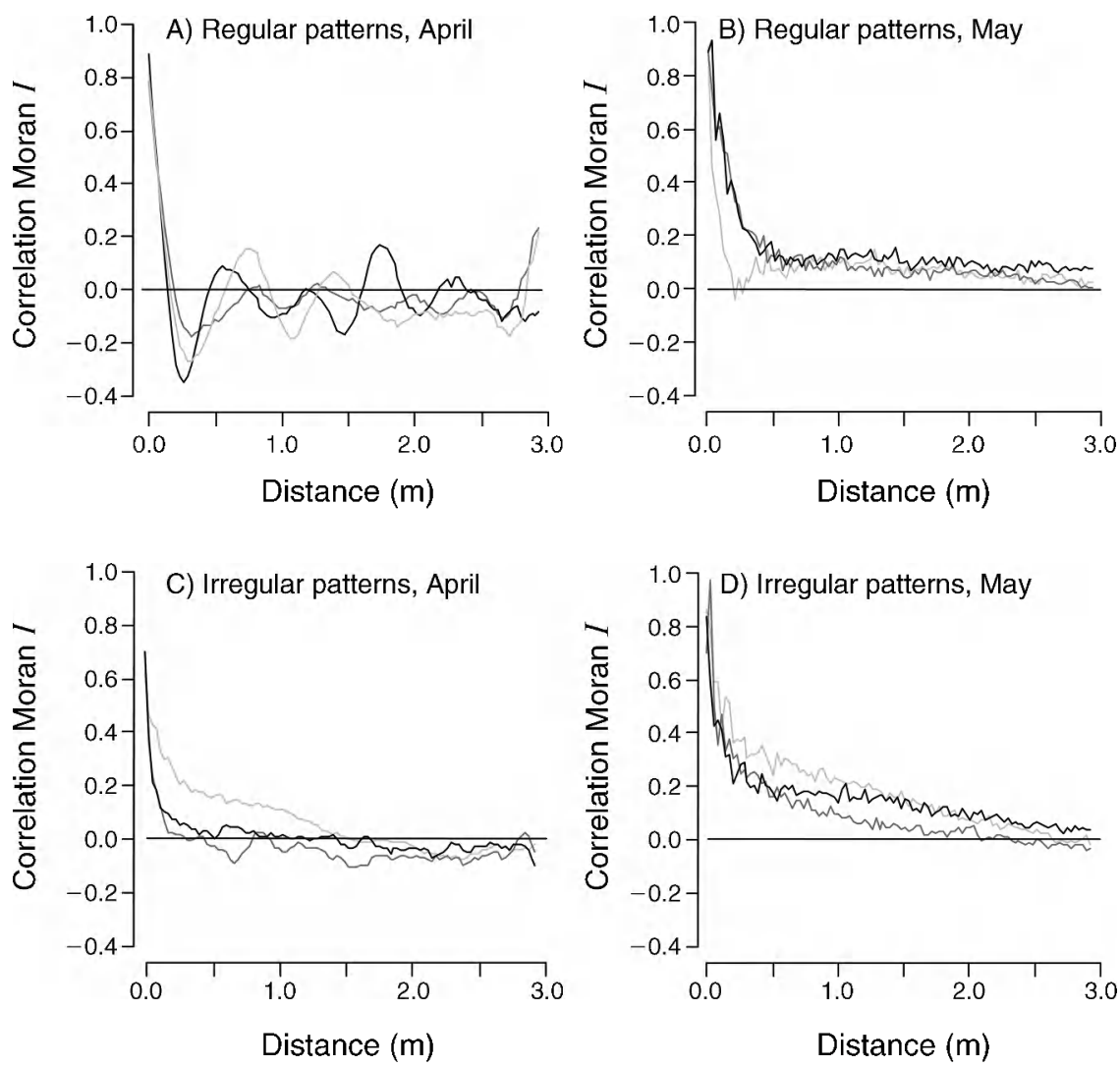

FIG. 3. Autocorrelation analysis of the diatom patterns with the Moran $I$ values for the regular patterns in (A) April and (B) May and irregular plots in (C) April and (D) May. The three different shades of lines indicate the three different plots.

ecosystems might provide information regarding the level of ecosystem degradation.

Most of our study area was covered by regular patterns of diatom biofilms due to a scale-dependent feedback between the diatoms and sedimentation (Weerman et al. 2010). With ongoing degradation, the scaledependent diatom-sediment feedback was lost, which resulted in homogenous flat sediment. Similar regularity of diatom patches (Weerman et al. 2010) and the seasonal shift from patterned to homogeneous mudflat have been described for different sites in other years (de Brouwer et al. 2000, Weerman et al. 2011). This change in topography co-occurred with the loss of regularity of the spatial patterns and associated changes in the shape of the distribution toward a power law. This was reflected by an increase in the scaling exponent and a decrease of the truncation parameter, clearly indicating a loss of the larger patches from the distribution as the ecosystem becomes more degraded and fragmented.

Changes in patch-size distribution in response to increased grazing intensity have been reported for both Mediterranean and Mongolian dry grasslands (Kéfi et al. 2007, Lin et al. 2010). Yet, increased grazing intensity effected patch-size distribution differently in our study compared to grassland ecosystems, as the above studies reported on an increase of truncation of the patch-size distribution with increasing grazing intensity (Kéfi et al. 2007, but see Maestre and Escudero 2009), while we report on a decrease. These seemingly opposing findings can be explained by differences in the type of spatial pattern, and the mechanism that underlies spatial selforganization in these systems. The reference state proposed in the study by Kéfi et al. (2007) is a power law, which becomes increasingly truncated as grazing intensity increases as large patches are progressively lost and fragmented. On intertidal mudflats, local feedback processes between diatom growth, water drainage and sedimentation generates clear regular patterns when undisturbed, characterized by strongly truncated patchsize distributions. When grazing intensity increases through the season, patches are fragmented by the direct and indirect effects of grazing (Weerman et al. 2011). As a result, truncation disappears, and patch-size distribution approaches a power law with increased grazing intensity. Hence, in the intertidal mudflat studied here, the transition to degradation was associated with a shift in the processes that govern pattern formation; from self-organized regular patterns, characterized by a dominant spatial scale, to irregular and fragmented patterns, characterized by patch sizes 

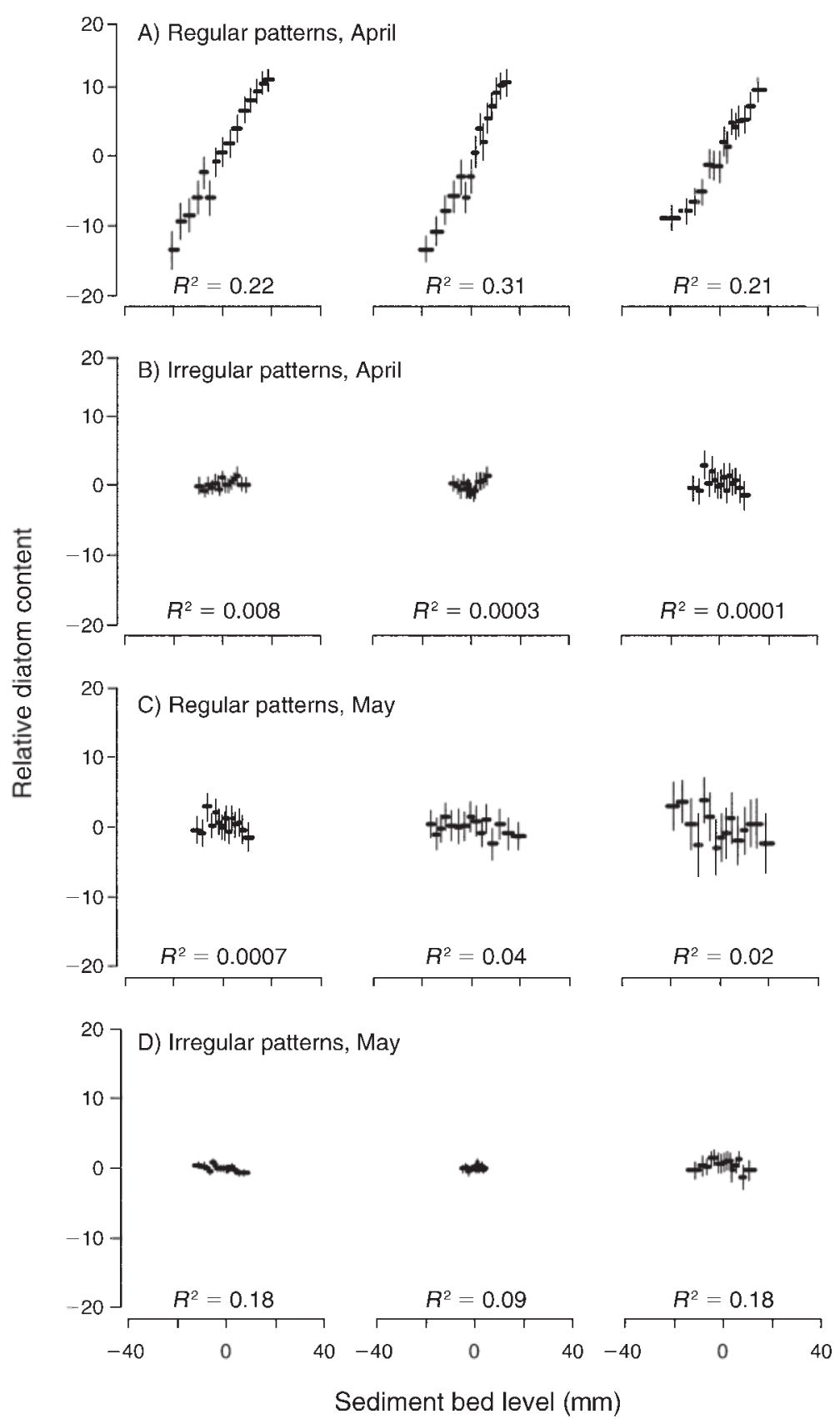

FIG. 4. The response of relative diatom abundance as a function of bed level. The data are split into 15 classes of the variables, with an equal number of observations in each class, and the plots show the mean value of each class and the $95 \%$ confidence intervals. Values are given for the adjusted $R^{2}$ of the model fit to the data.

lacking any characteristic scale. This comparison highlights the context dependency of the use of spatial patterns as indicators of degradation in ecosystems.

Although several studies have argued that spatial patterns could be used to predict the onset of degradation and catastrophic collapse (Rietkerk et al. 2004, Kéfi et al. 2007), the use of patch-size distribution as a monitoring tool to gauge ecosystem degradation levels is still in its infancy, as shown by the recent discussions in the literature (Kéfi et al. 2007, 2010, Maestre and Escudero 2009, 2010, Pueyo 2011). One of the main challenges is to develop proper statistics and robust ways of evaluating the truncation of the distributions (Pueyo 2011). Our results contribute to this discussion and suggest that the changes in spatial patterns that can be expected with ongoing degradation 

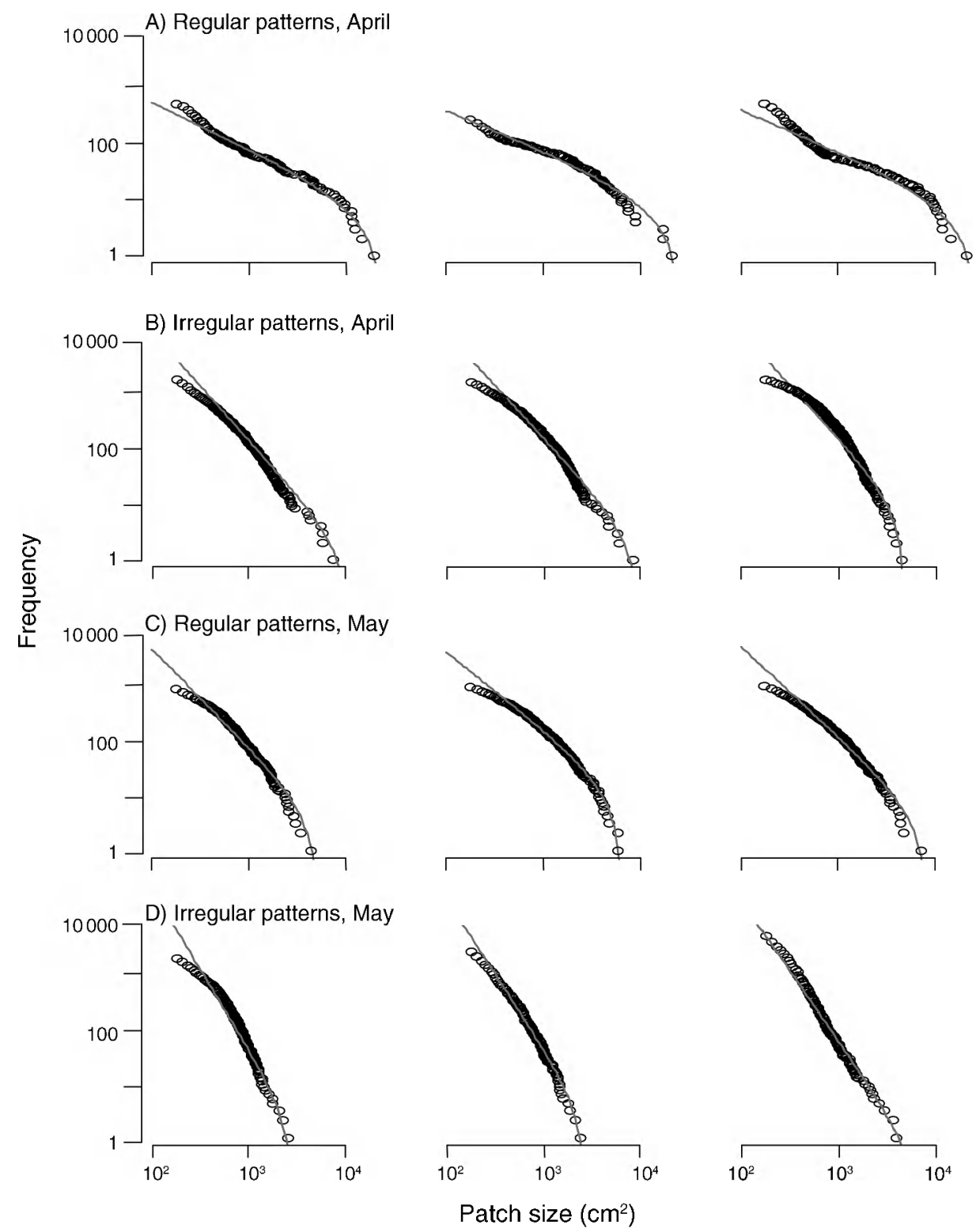

FIG. 5. Patch-size distribution of diatom patches from three replicas of each location and month. The $x$-axis represents the patch size $\left(\mathrm{m}^{2}\right)$, and the $y$-axis represents frequency of patch size. Circles are observed values, and the gray line is the fitted model.

are determined by the type of patterns that characterizes the undisturbed ecosystem, and hence by the ecological mechanisms underlying the patterns. Understanding the "pattern attractor," i.e., the type of pattern that can be expected under undisturbed conditions (e.g., regular or irregular, self-organized or not), is essential before predictions on how degradation can affect the patchsize distribution can be safely made. Therefore, we argue that, at the very least, the presence of self-organization processes in any spatially heterogeneous ecosystem needs to be empirically verified, before spatial patterns can be used as indicator for ongoing or imminent degradation.
The loss of cover fraction of vegetation, or of other organisms, is the most direct way to assess the degree of deterioration of ecosystems (Reynolds et al. 2007, Maestre and Escudero 2009, 2010). However, ecosystems with strong feedback mechanisms can respond nonlinearly, involving tipping points, to gradually increased stress (Rietkerk et al. 2004, Scheffer et al. 2009). In those cases, the cover fraction at which a tipping point occurs is unknown, and cover fraction alone is not sufficient to indicate the proximity of a tipping point in the ecosystem (Kéfi et al. 2010). Our study demonstrates that the change of the scaling exponent reflected increasing fragmentation by grazing 

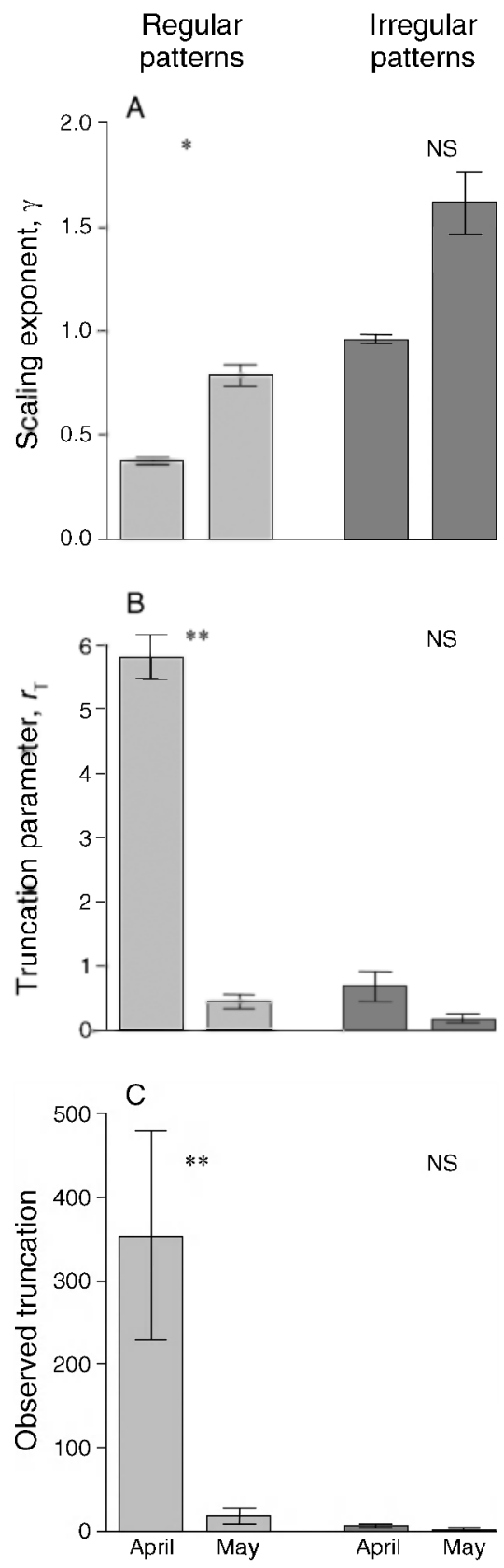

FIG. 6. Fitted parameters from our patch-size distribution analysis describing the (A) scaling exponent $\gamma_{\text {. (B) truncation }}$ parameter $r_{\mathrm{T}}$, and (C) observed truncation for regular and irregular patterns in April and May. Light gray bars represent the regular spacing patterns, and dark gray bars represent the irregular patterns $(n=3$; error bars indicate $\pm \mathrm{SE}$ ).

$* P<0.05 ; * * P<0.01 ; * * * P<0.001$; NS, not significant.

activity, as the scaling exponent $\gamma$ increases with level of degradation. Nevertheless, increase in the scaling exponent alone provides no indication of the breakdown of key feedback processes, nor could it signal a possible catastrophic response. This is underlined by an equally strong change in the scaling exponent found in plots on the mudflat that only contained irregular diatom patches. In those plots, the scale-dependent feedback was less important as a structuring force of the diatom community. Nonetheless, the scaling exponent increases with increased disturbance levels, suggesting that the scaling exponent match the level of fragmentation. So, in this study, the level of truncation is an indication of feedback strength, whereas the scaling exponent reflects level of fragmentation.

Other indicators for possible nonlinear responses, like increased spatial autocorrelation and variance in proximity of a tipping point have recently been proposed (Guttal and Jayaprakash 2009, Dakos et al. 2010, Carpenter et al. 2011). The autocorrelation in our study drops during degradation, when the regularity of the patterns becomes weaker. This is in line with a recent model study by Dakos et al. (2011) where they found that degradation in regular self-organized patterns failed to show increase in rising variance and autocorrelation. The combined results of the theoretical study by Dakos et al. (2011) and the results in our empirical study imply that the spatial autocorrelation solely is not a reliable indicator in predicting regime shifts in regular selforganized patterns. This suggests that a set of indicators might be more powerful in predicting regime shifts than a single indicator (Kéfi et al. 2011). Therefore, we suggest that, for regular self-organized patterns, a combination of the observed truncation and autocorrelation, both signatures of regularity, are the most reliable assessment of ecosystem health.

Concluding, our results show that changes in the structure of spatial patterns can provide important clues about the level of degradation in self-organized ecosystems. However, the sequence in which patterns change with ongoing degradation, as well as the type of changes, depends on the underling structuring mechanisms that generate these patterns, e.g., whether they result from feedback leading to self-organization, and what type of self-organization. We suggest that spatial indicators take their full value when they are combined with measures of ecological mechanisms. We can test and compare different measures of the spatial structure (patch-size distributions and many others such as spatial autocorrelation, spatial variance, and mean patch size) and we can evaluate those along transitions (in experimental systems and models). In parallel to that, we can measure the feedback mechanisms, such as we describe in this paper. Therefore, we propose that, before spatial patterns can be used to predict degradation in ecosystems, it is important to (1) resolve the mechanisms that underlie both the formation and degradation of spatial patterns, (2) develop a description of the pattern attractors that describe the expected patterns in undisturbed conditions and how it changes when disturbed, and (3) develop indicators of both the structure of the spatial patterns and of the strength of the underlying feedback mechanisms, before a reliable assessment of ecosystem status can be made. Only when fulfilling 


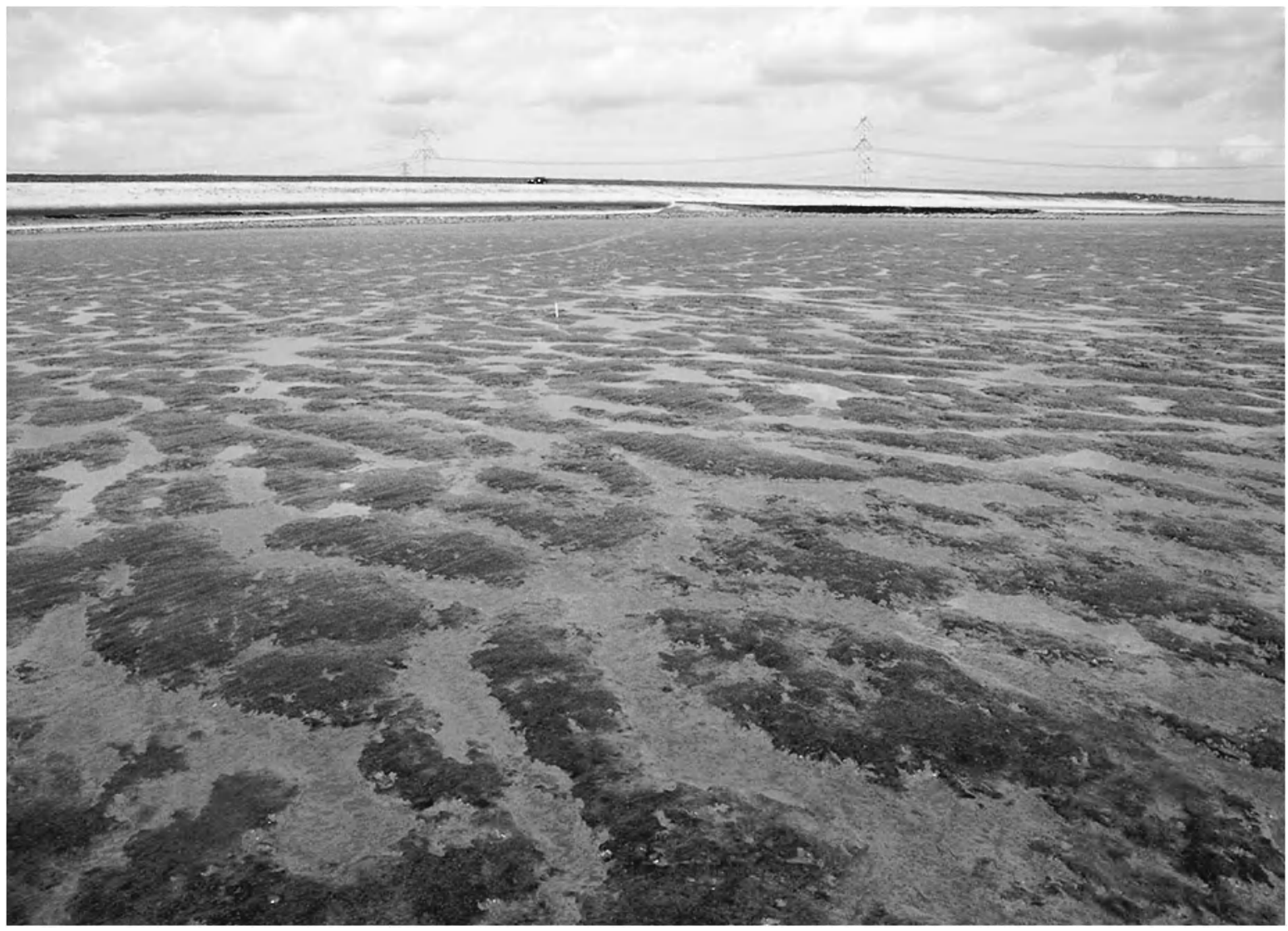

Plate 1. Regularly patterned landscape of diatom-covered hummocks alternating with water-filled hummocks at the Kapellebank. The Netherlands. Photo credit: E. J. Weerman.

above three criteria, spatial structure can become a promising indicator to address the proximity of a nonlinear response toward complete degradation.

\section{ACKNOWLEDGMENTS}

The authors thank Nico de Regge for help with the 3-D laser scanner equipment. and Jos van Soelen and Lennart van IJzerloo for their valuable help in the field. The work of J. van Belzen was financed by the EU project THESEUS. David Alonso, Fernando T. Maestre, and Simon Thrush provided valuable comments on earlier drafts of the manuscript. This is NIOO publication number 5118

\section{Literature Cited}

Barbier, N., P. Couteron, R. Lefever, V. Deblauwe, and O. Lejeune. 2008. Spatial decoupling of facilitation and competition at the origin of gapped vegetation patterns. Ecology 89:1521-1531.

Blanchard, G. F.. D. M. Paterson. L. J. Stal. P. Richard, R. Galois, V. Huet, J. Kelly, C. Honeywill, J. de Brouwer, K. Dyer. M. Christie, and M. Seguignes. 2000. The effect of geomorphological structures on potential biostabilisation by microphytobenthos on intertidal mudflats. Continental Shelf Research 20:1243-1256.

Burroughs, S. M.. and S. F. Tebbens. 2001. Upper-truncated power laws in natural systems. Pure and Applied Geophysics 158:741-757.

Carpenter. S. R.. J. J. Cole, M. R. Pace. R. Batt. W. A. Brock, T. Cline, J. Coloso, J. R. Hodgson, J. F. Kitchell, D. A.
Seekell, L. Smith, and B. Weidel. 2011. Early warnings of regime shifts: a whole-ecosystem experiment. Science 332:1079-1082.

Christie, M. C.. K. R. Dyer, G. Blanchard, A. Cramp, H. J. Mitchener, and D. M. Paterson. 2000. Temporal and spatial distributions of moisture and organic contents across a macro-tidal mudflat. Pages 1219-1241.

Dakos, V., S. Kéfi, M. Rietkerk, E. H. Van Nes, and M. Scheffer. 2011. Slowing down in spatially patterned ecosystems at the brink of collapse. American Naturalist 177:E153E166.

Dakos, V., E. H. Van Nes, R. Donangelo, H. Fort, and M. Scheffer. 2010. Spatial correlation as leading indicator of catastrophic shifts. Theoretical Ecology 3:163-174.

de Brouwer. J. F. C.. S. Bjelic. E. de Deckere. and L. J. Stal. 2000. Interplay between biology and sedimentology in a mudflat (Biezelingse Ham. Westerschelde. The Netherlands). Continental Shelf Research 20:1159-1177.

Guttal, V.. and C. Jayaprakash. 2009. Spatial variance and spatial skewness: leading indicators of regime shifts in spatial ecological systems. Theoretical Ecology 2:3-12.

Hiemstra, C. A., G. E. Liston, and W. A. Reiners. 2002. Snow redistribution by wind and interactions with vegetation at upper treeline in the Medicine Bow Mountains, Wyoming. USA. Arctic Antarctic and Alpine Research 34:262-273.

Kéfi. S., C. L. Alados, R. C. G. Chaves, Y. Pueyo, and M. Rietkerk. 2010. Is the patch size distribution of vegetation a suitable indicator of desertification processes? Comment. Ecology 91:3739-3742. 
Kéfi, S., M. Rietkerk, C. L. Alados, Y. Pueyo, V. P. Papanastasis, A. ElAich. and P. C. de Ruiter. 2007. Spatial vegetation patterns and imminent desertification in Mediterranean arid ecosystems. Nature 449:213-U215.

Kéfi, S., M. Rietkerk, M. Roy, A. Franc, P. C. De Ruiter, and M. Pascual. 2011. Robust scaling in ecosystems and the meltdown of patch size distributions before extinction. Ecology Letters 14:29-35.

Lanuru, M., R. Riethmuller, C. van Bernem, and K. Heymann. 2007. The effect of bedforms (crest and trough systems) on sediment erodibility on a back-barrier tidal flat of the East Frisian Wadden Sea. Germany. Estuarine Coastal and Shelf Science 72:603-614.

Larsen, L. G., J. W. Harvey, and J. P. Crimaldi. 2007. A delicate balance: ecohydrological feedbacks governing landscape morphology in a lotic peatland. Ecological Monographs 77:591-614.

Legendre, P.. and L. Legendre. 1998. Numerical ecology. Elsevier, Amsterdam, The Netherlands.

Le Hir, P., W. Roberts, O. Cazaillet, M. Christie, P. Bassoullet, and C. Bacher. 2000. Characterization of intertidal flat hydrodynamics. Continental Shelf Research 20:1433-1459.

Lin, Y., G. Han, M. Zhao, and S. X. Chang. 2010. Spatial vegetation patterns as early signs of desertification: a case study of a desert steppe in Inner Mongolia, China. Landscape Ecology 25:1519-1527.

Maestre, F. T., and A. Escudero. 2009. Is the patch size distribution of vegetation a suitable indicator of desertification processes? Ecology 90:1729-1735.

Maestre. F. T.. and A. Escudero. 2010. Is the patch size distribution of vegetation a suitable indicator of desertification processes? Reply. Ecology 91:3742-3745.

Pascual, M., and F. Guichard. 2005. Criticality and disturbance in spatial ecological systems. Trends in Ecology and Evolution 20:88-95.

Paterson, D. M.. T. J. Tolhurst, J. A. Kelly, C. Honeywill, E. de Deckere, V. Huet, S. A. Shayler, K. S. Black, J. de Brouwer, and I. Davidson. 2000. Variations in sediment properties, Skeffling mudflat, Humber Estuary, UK. Continental Shelf Research 20:1373-1396.
Pueyo, S. 2011. Desertification and power laws. Landscape Ecology 26:305-309.

Reynolds. J. F.. et al. 2007. Global desertification: building a science for dryland development. Science 316:847-851.

Rietkerk, M., S. C. Dekker, P. C. de Ruiter, and J. van de Koppel. 2004. Self-organized patchiness and catastrophic shifts in ecosystems. Science 305:1926-1929.

Rietkerk. M., and J. Van de Koppel. 2008. Regular pattern formation in real ecosystems. Trends in Ecology and Evolution 23:169-175.

Saco, P. M., G. R. Willgoose, and G. R. Hancock. 2007. Ecogeomorphology of banded vegetation patterns in arid and semi-arid regions. Hydrology and Earth System Sciences 11:1717-1730.

Scheffer, M., J. Bascompte, W. A. Brock, V. Brovkin. S. R. Carpenter, V. Dakos. H. Held. E. H. van Nes. M. Rietkerk. and G. Sugihara. 2009. Early-warning signals for critical transitions. Nature 461:53-59.

Soetaert. K., and P. M. J. Herman. 2009. A practical guide to ecological modelling. Using $\mathrm{R}$ as a simulation platform. Springer, Berlin. Germany.

Temmerman, S.. T. J. Bouma, J. Van de Koppel, D. D. Van der Wal, M. B. De Vries, and P. M. J. Herman. 2007. Vegetation causes channel erosion in a tidal landscape. Geology 35:631634.

van Wesenbeeck. B. K.. J. van de Koppel. P. M. J. Herman. and T. J. Bouma. 2008. Does scale-dependent feedback explain spatial complexity in salt-marsh ecosystems? Oikos $117: 152-159$.

von Hardenberg. J.. A. Y. Kletter. H. Yizhaq. J. Nathan. and E. Meron. 2010. Periodic versus scale-free patterns in dryland vegetation. Proceedings of the Royal Society B 277:17711776.

Weerman. E. J., P. M. J. Herman, and J. Van de Koppel. 2011. Top-down control regulates self-organization on a patterned intertidal flat. Ecology 92:487-495.

Weerman, E. J., J. van de Koppel, M. B. Eppinga, F. Montserrat, Q. X. Liu, and P. M. J. Herman. 2010. Spatial self-organization on intertidal mudflats through biophysical stress divergence. American Naturalist 176:E15-E32.

\section{Supplemental Material}

Appendix A

Detailed description of the study site and methods (Ecological Archives E093-054-A1).

\section{Appendix B}

Aerial photographs of our plots and their binary images after imaging processing (Ecological Archives E093-054-A2). 